\title{
Van der Waals cascade in supercritical turbulence near a critical point
}

\author{
Tomohiro Tanogami 1 a and Shin-ichi Sasa ${ }^{(1)}$ \\ Department of Physics, Kyoto University, Kyoto 606-8502, Japan
}

(Received 27 January 2021; accepted 7 July 2021; published 30 July 2021)

\begin{abstract}
We investigate a quite strong turbulence in a supercritical fluid near a gas-liquid critical point. Specifically, we consider a case in which the Kolmogorov scale is much smaller than the equilibrium correlation length $\xi$. Although equilibrium critical fluctuations are destroyed by turbulence, $\xi$ still provides a crossover length scale between two types of energy cascade. At scales much larger than $\xi$, the Richardson cascade becomes dominant, whereas at scales much smaller than $\xi$, another type of cascade, which we call the van der Waals cascade, is induced by density fluctuations. Experimental conditions required to observe the van der Waals cascade are also discussed.
\end{abstract}

DOI: 10.1103/PhysRevResearch.3.L032027

Introduction. Nonlinearity, which appears ubiquitously in a broad range of phenomena, causes inevitable interference between widely separated time and space scales. One of the most extreme examples is found in fully developed turbulence. In turbulence, the kinetic energy is transferred conservatively and continuously from large to small scales in the so-called inertial range [1]. The mechanism of this remarkable phenomenon, the Richardson cascade, was intuitively explained by Richardson's depiction of a large vortex splitting into smaller vortices [2]. As a consequence of the energy transfer, the kinetic energy spectrum exhibits a powerlaw behavior-the Kolmogorov spectrum [3,4]. The kinetic energy transported to small scales is dissipated at the Kolmogorov scale, where viscosity begins to predominate, so that the Richardson cascade is inevitably cut off at the Kolmogorov scale. The important point here is that in standard cases, the Kolmogorov scale is overwhelmingly larger than the microscopic length scales, such as the molecular mean free path [1]. Therefore the cascade never reaches microscopic length scales in these cases.

Another notable instance in which nonlinearity causes strong interference between widely separated scales is found in critical phenomena. As an example, in the vicinity of a gas-liquid critical point, the correlation length of equilibrium density fluctuations $\xi$ reaches a macroscopic order of magnitude $[5,6]$. We here consider the strong turbulent regime of a supercritical fluid near a critical point in which $\xi$ is much larger than the Kolmogorov scale. Even for such strong turbulence, $\xi$ still provides a length scale at which the stress induced by density fluctuations is comparable to the momentum flux. In this case density fluctuations are driven by

Published by the American Physical Society under the terms of the Creative Commons Attribution 4.0 International license. Further distribution of this work must maintain attribution to the author(s) and the published article's title, journal citation, and DOI. turbulence so that the equilibrium critical fluctuations are destroyed. We then ask how the Richardson cascade is modified by density fluctuations in the turbulence near a critical point. Although turbulence in supercritical fluids has been studied over the past few decades, previous studies have focused on cases in which the Kolmogorov scale is larger than $\xi$ [7-10].

We answer the above question by studying hydrodynamic equations including density fluctuations. Specifically, we include a density gradient contribution to the entropy functional to describe the effects of density fluctuations. Such a formulation that takes into account gradient contributions was originally proposed in the pioneering work of van der Waals [11], who introduced a gradient term in the Helmholtz free energy density to describe a gas-liquid interface, and the formulation has been widely used in statistical mechanics since the publication of seminal papers by Ginzburg and Landau for type-I superconductors [12] and by Cahn and Hilliard for binary alloys [13]. Following the van der Waals theory, Korteweg proposed hydrodynamic equations that contain the van der Waals stress (vdW stress) arising from the density gradient $[14,15]$, and Onuki generalized the theory by including the gradient contribution to both entropy and energy functionals $[16,17]$.

In this study we analyze the model using a phenomenological approach based on the Onsager "ideal turbulence" theory [18-20]. The Onsager theory describes the essence of turbulent behavior, such as the Richardson cascade and energy dissipation in the absence of viscosity, the so-called anomalous dissipation [21]. Although the Onsager theory involves sophisticated mathematical concepts such as weak solutions, it also provides a phenomenological perspective on the relation between cascades and the singularity of the velocity field. This theory has been recently extended to various turbulent phenomena, such as compressible turbulence [22-27] and plasma turbulence [28], and has also been intensively studied from a deep mathematical point of view related to convex integration [29-33].

In this Letter we show that supercritical turbulence near a critical point exhibits the Richardson cascade and another 
type of cascade-the van der Waals cascade-induced by the van der Waals stress. First, we derive the scale independence of the scale-to-scale kinetic energy flux in the "inertial range." Second, we show the possibility of the existence of the van der Waals cascade. Furthermore, we consider the experimental conditions required to observe such van der Waals turbulence, which exhibits both the Richardson and van der Waals cascades.

Setup. Let $\rho$ be the mass density, $\mathbf{v}$ be the fluid velocity, and $u$ be the internal energy density. For simplicity, we assume that a fluid is confined in a cube $\Omega=[0, \mathcal{L}]^{3}$ with periodic boundary conditions. We further assume that there is no vacuum region; i.e., $\rho(\mathbf{x}, t)>0$ for all $\mathbf{x} \in \Omega$ and $t \in \mathbb{R}$. Following the van der Waals theory, we include a gradient contribution to the entropy functional to describe enhanced density fluctuations near a critical point $[11,16,17,34,35]$ :

$$
\mathcal{S}([u],[\rho])=\int_{\Omega} d^{3} \mathbf{x}\left(s(u, \rho)+\frac{c(\rho)}{2}|\nabla \rho|^{2}\right),
$$

where $([u],[\rho]):=(u(\mathbf{x}), \rho(\mathbf{x}))_{\mathbf{x} \in \Omega}, s(u, \rho)$ denotes the entropy density, and $c(\rho) \leqslant 0$ is the capillary coefficient. In the following discussion, we consider the case in which the capillary coefficient is a sufficiently smooth function of $\rho$, e.g., $c(\rho)=$ const [17]. Through thermodynamic relations, the temperature $T(u, \rho)$ and pressure tensor $\mathbf{P}$ are determined from (1) [36]:

$$
\mathbf{P}=p \mathbf{I}+\mathbf{\Sigma},
$$

where $p(u, \rho)$ denotes the pressure defined by $s(u, \rho), \mathbf{I}$ is the unit tensor, and $\boldsymbol{\Sigma}$ is the $v d W$ stress tensor, which arises from the gradient contribution and is defined by

$$
\boldsymbol{\Sigma}:=\left(T c \rho \Delta \rho+\frac{1}{2} T c^{\prime} \rho|\nabla \rho|^{2}+\frac{1}{2} T c|\nabla \rho|^{2}\right) \mathbf{I}-T c \nabla \rho \nabla \rho .
$$

The time evolution of the densities of mass $\rho$, momentum $\rho \mathbf{v}$, and total energy $\rho|\mathbf{v}|^{2} / 2+u$ is then governed by the NavierStokes-Korteweg equations [37-39]:

$$
\begin{gathered}
\partial_{t} \rho+\nabla \cdot(\rho \mathbf{v})=0 \\
\partial_{t}(\rho \mathbf{v})+\nabla \cdot(\rho \mathbf{v v}+\mathbf{P}+\boldsymbol{\sigma})=\mathbf{f} \\
\partial_{t}\left(u+\frac{1}{2} \rho|\mathbf{v}|^{2}\right)+\nabla \cdot\left\{\left[\left(u+\frac{1}{2} \rho|\mathbf{v}|^{2}\right) \mathbf{I}+\mathbf{P}+\boldsymbol{\sigma}\right] \cdot \mathbf{v},\right. \\
-\lambda \nabla T\}=\mathbf{v} \cdot \mathbf{f},
\end{gathered}
$$

where $\mathbf{f}$ denotes an external force acting at large scales $\sim L, \lambda$ is the thermal conductivity, and $\sigma$ is the viscous stress tensor of the form

$$
\sigma_{i j}=-\mu\left(\partial_{i} v_{j}+\partial_{j} v_{i}-\frac{2}{3} \delta_{i j} \nabla \cdot \mathbf{v}\right)-\zeta \delta_{i j} \nabla \cdot \mathbf{v} .
$$

Here $\mu$ and $\zeta$ are the shear and bulk viscosity coefficients, respectively. We assume that the viscous effect is sufficiently weak for the Kolmogorov scale to be sufficiently smaller than any other length scales.

In the following, (4)-(6) are applied even to scales smaller than the equilibrium correlation length. Strictly speaking, dynamics at such scales should be described within the framework of fluctuating hydrodynamics [40]. In a turbulent regime, however, the equilibrium correlation may be cut off, and the noise terms may be irrelevant for energy transfer. We therefore assume that (4)-(6) are sufficient for our phenomenological argument.

Characteristic length scales. Owing to the effect of the gradient contribution, several characteristic length scales that are not relevant in ordinary fluid turbulence become important. Let $\rho_{0}:=\langle\rho\rangle, c_{0}:=c\left(\rho_{0}\right)$, and $T_{0}:=\langle T\rangle$ be the typical density, capillary coefficient, and temperature, respectively, where $\langle\cdot\rangle$ denotes the volume average $\int_{\Omega} \cdot d^{3} \mathbf{x} / \mathcal{L}^{3}$. In addition, let $v_{0}:=\left(\rho_{0} K_{T_{0}}\right)^{-1 / 2}$ be a velocity characterized by the isothermal compressibility $K_{T_{0}}:=\rho_{0}^{-1} \partial \rho\left(T_{0}, p\right) / \partial p$, which is zero at a critical point. One of the most crucial length scales is the correlation length of equilibrium density fluctuations,

$$
\xi=\frac{\sqrt{T_{0}\left|c_{0}\right| \rho_{0}}}{v_{0}}
$$

which is expressed by the capillary coefficient $c(\rho)$ and parameters in the entropy density $s(u, \rho)$ [41]. The important point here is that even for strong turbulence, $\xi$ still provides a characteristic length scale at which the vdW stress $\boldsymbol{\Sigma}$ and momentum flux $\rho \mathbf{v v}$ are comparable. Let $\ell_{c}$ be such a length scale. Using an estimation that $\rho \mathbf{v v} \sim \rho_{0} v_{0}^{2}$ and $\boldsymbol{\Sigma} \sim$ $T_{0}\left|c_{0}\right| \rho_{0}^{2} / \ell_{c}^{2}$, we obtain

$$
\ell_{c} \sim \xi
$$

Note that $\boldsymbol{\Sigma}$ can be appreciable at small scales because it contains higher-order spatial derivatives. Therefore, at scales $\gg \ell_{c}$, the effect of the $\mathrm{vdW}$ stress is small compared with the momentum flux, whereas at scales $\ll \ell_{c}$, the vdW stress becomes relevant. This observation implies the possibility of the van der Waals cascade, induced by the vdW stress, at scales $\ll \ell_{c}$.

We attempt to seek other characteristic length scales by noting the local kinetic energy balance equation:

$$
\begin{aligned}
& \partial_{t}\left(\frac{1}{2} \rho|\mathbf{v}|^{2}\right)+\nabla \cdot\left[\left(\frac{1}{2} \rho|\mathbf{v}|^{2} \mathbf{I}+\mathbf{P}+\boldsymbol{\sigma}\right) \cdot \mathbf{v}\right] \\
& =p \nabla \cdot \mathbf{v}+\mathbf{\Sigma}: \nabla \mathbf{v}+\boldsymbol{\sigma}: \nabla \mathbf{v}+\mathbf{v} \cdot \mathbf{f} .
\end{aligned}
$$

The first term on the right-hand side of $(10),-p \nabla \cdot \mathbf{v}$, is the pressure dilatation, which represents the conversion of kinetic energy into internal energy and vice versa. Recent numerical simulations $[42,43]$ suggest that there is a characteristic length scale $\ell_{\text {large }}$ such that the contribution to the global pressure dilatation $\langle-p \nabla \cdot \mathbf{v}\rangle$ from scales $\gg \ell_{\text {large }}$ is dominant, whereas the contribution from scales $\ll \ell_{\text {large }}$ is negligible. The second term on the right-hand side of (10), $-\Sigma: \nabla \mathbf{v}$, which we call the $v d W$-stress-strain, arises because of the gradient contribution. It also represents the conversion between kinetic and internal energy. Because both the vdW stress $\boldsymbol{\Sigma}$ and strain $\nabla \mathbf{v}$ change rapidly in space, there may be a characteristic length scale $\ell_{\text {small }}$ such that the contribution to the global vdW-stress-strain $\langle-\Sigma: \nabla \mathbf{v}\rangle$ from scales $\gg \ell_{\text {small }}$ is negligible, whereas the contribution from scales $\ll \ell_{\text {small }}$ is dominant. In the following, we assume the existence of the intermediate asymptotic limit $\ell_{\text {small }} \ll \ell \ll \ell_{\text {large }}$ that satisfies $\ell_{\text {small }} \ll \ell_{c}$ and $\ell_{c} \ll \ell_{\text {large }}$.

Main result. Let $Q_{\ell}^{\text {flux }}$ be the scale-to-scale kinetic energy flux that represents the energy transfer from scales $>\ell$ to 
scales $<\ell$ :

$$
Q_{\ell}^{\text {flux }}:=\Pi_{\ell}+\Lambda_{\ell}^{(p)}+\Lambda_{\ell}^{(\Sigma)},
$$

where $\Pi_{\ell}$ is deformation work [44], which corresponds to the energy flux of the Richardson cascade, $\Lambda_{\ell}^{(p)}$ is baropycnal work [25,45], which arises because of compressibility, and $\Lambda_{\ell}^{(\Sigma)}$ is capillary work, which arises because of the gradient contribution. (Precise definitions are given below.) These three terms represent the energy transfer due to the momentum flux $\rho \mathbf{v v}$, pressure $p$, and vdW stress $\boldsymbol{\Sigma}$, respectively. The first main claim of this paper concerns the most crucial property of the energy cascade; i.e., in a steady state with a constant mean kinetic energy, $\left\langle Q_{\ell}^{\text {flux }}\right\rangle$ becomes scale independent in the "inertial range" $\ell_{\text {small }} \ll \ell \ll \ell_{\text {large }}$ :

$$
\left\langle Q_{\ell}^{\text {flux }}\right\rangle \approx \epsilon_{\text {eff }},
$$

where $\epsilon_{\text {eff }}:=\langle p \nabla \cdot \mathbf{v}\rangle+\langle\mathbf{v} \cdot \mathbf{f}\rangle$ denotes the effective energy injection rate, which is scale independent. We emphasize that because $Q_{\ell}^{\text {flux }}$ can be expressed in terms of field increments $\mathbf{v}(\mathbf{x}+\mathbf{r})-\mathbf{v}(\mathbf{x})$ and $\rho(\mathbf{x}+\mathbf{r})-\rho(\mathbf{x})$, the relation (12) plays the same role as Kolmogorov's 4/5 law [4].

The second main result of this paper is the prediction of the van der Waals cascade. In the range of $\ell_{c} \ll \ell \ll \ell_{\text {large }}$, the Richardson cascade, induced by the momentum flux, becomes dominant, whereas in the range of $\ell_{\text {small }} \ll \ell \ll \ell_{c}$, the van der Waals cascade, induced by vdW stress, develops [46]:

$$
\begin{gathered}
\left\langle\Lambda_{\ell}^{(\Sigma)}\right\rangle \ll\left\langle\Pi_{\ell}\right\rangle \approx \epsilon_{\text {eff }} \quad \text { for } \quad \ell_{c} \ll \ell \ll \ell_{\text {large }}, \\
\left\langle\Pi_{\ell}\right\rangle \ll\left\langle\Lambda_{\ell}^{(\Sigma)}\right\rangle \approx \epsilon_{\text {eff }} \text { for } \quad \ell_{\text {small }} \ll \ell \ll \ell_{c} .
\end{gathered}
$$

Correspondingly, the velocity power spectrum exhibits a power-law behavior: $k^{-5 / 3}$ for $\ell_{\text {large }}^{-1} \ll k \ll \ell_{c}^{-1}$ and $k^{-3}$ for $\ell_{c}^{-1} \ll k \ll \ell_{\text {small }}^{-1}$.

Suggested experiments. We consider the experimental conditions required for observing the van der Waals cascade. In the study of critical phenomena, $\mathrm{CO}_{2}$ has been widely used because its critical state occurs under readily realized experimental conditions $\left(T_{c}=304.13 \mathrm{~K}, p_{c}=7.3773 \mathrm{MPa}\right.$, $\rho_{c}=0.4678 \mathrm{~g} \mathrm{~cm}^{-3}$ ) $[47,48]$. In this case the shear viscosity $\mu$ takes a value around $3.5 \times 10^{-4} \mathrm{~g} \mathrm{~cm}^{-1} \mathrm{~s}^{-1}[10,47,49]$. We first estimate the Kolmogorov scale $\ell_{d}$, which can be estimated in terms of $\mu, \rho_{c}, L$, and $v_{\text {rms }}:=\sqrt{\left\langle|\mathbf{v}|^{2}\right\rangle}$ as

$$
\ell_{d} \sim L^{1 / 4}\left(\frac{\mu}{\rho_{c} v_{\mathrm{rms}}}\right)^{3 / 4} .
$$

If we achieve a quite strong turbulent regime, in which $\operatorname{Re} \sim$ $10^{5}$ (e.g., $v_{\text {rms }} \sim 100 \mathrm{~m} / \mathrm{s}$ and $L \sim 0.1 \mathrm{~m}$ ), the Kolmogorov scale is $\sim 800 \AA$. Therefore if one can reach the vicinity of the critical point such that the correlation length is at least $\sim 10000 \AA$, it may be possible to verify our predictions by measuring the velocity field using hot-wire anemometry or laser Doppler velocimetry. To achieve a correlation length of that magnitude, we must control the system with an accuracy of at least $T-T_{c} \sim 10^{-4} \mathrm{~K}$ because $\xi \approx \xi_{0} \epsilon^{-v}$, where $\xi_{0}=1.5 \AA, \epsilon:=\left(T-T_{c}\right) / T_{c}$, and $v=0.630[47,49]$.

Derivation of the main result. We study the properties of kinetic energy transfer across scales using a coarse-graining approach that can resolve turbulent fields both in scale and in space. For any field $\mathbf{a}(\mathbf{x})$, we define a coarse-grained field at length scale $\ell$ as

$$
\overline{\mathbf{a}}_{\ell}(\mathbf{x}):=\int_{\Omega} d^{3} \mathbf{r} G_{\ell}(\mathbf{r}) \mathbf{a}(\mathbf{x}+\mathbf{r}),
$$

where $G: \Omega \rightarrow[0, \infty)$ is a smooth symmetric function supported in the open unit ball with $\int_{\Omega} G=1$, and $G_{\ell}(\mathbf{r}):=$ $\ell^{-3} G(\mathbf{r} / \ell)$ is the rescaling defined for each $\ell>0$. By coarse graining (4) and (5), we can write the coarse-grained kinetic energy balance equation

$$
\begin{aligned}
\partial_{t}\left(\frac{1}{2} \bar{\rho}_{\ell}\left|\tilde{\mathbf{v}}_{\ell}\right|^{2}\right)+\nabla \cdot \mathbf{J}_{\ell}= & \bar{p}_{\ell} \nabla \cdot \overline{\mathbf{v}}_{\ell}+\overline{\mathbf{\Sigma}}_{\ell}: \nabla \overline{\mathbf{v}}_{\ell} \\
& -Q_{\ell}^{\text {flux }}-D_{\ell}+\epsilon_{\ell}^{\text {in }},
\end{aligned}
$$

where we introduce the density-weighted coarse-grained velocity $\tilde{\mathbf{v}}_{\ell}:=\overline{(\rho \mathbf{v})}_{\ell} / \bar{\rho}_{\ell}$ to reduce the number of additional cumulant terms and to obtain a simple physical interpretation. Here, $\epsilon_{\ell}^{\mathrm{in}}:=\tilde{\mathbf{v}}_{\ell} \cdot \overline{\mathbf{f}}_{\ell}$ denotes the energy injection rate due to external stirring at scale $\ell, D_{\ell}:=-\nabla \tilde{\mathbf{v}}_{\ell}: \overline{\boldsymbol{\sigma}}_{\ell}$ denotes the viscous dissipation acting at scale $\ell$, and $\mathbf{J}_{\ell}$ represents the spatial transport of large-scale kinetic energy, which does not contribute to the energy transfer across scales. The first two terms on the right-hand side of (16), $-\bar{p}_{\ell} \nabla \cdot \overline{\mathbf{v}}_{\ell}$ and $-\overline{\boldsymbol{\Sigma}}_{\ell}: \nabla \overline{\mathbf{v}}_{\ell}$, are the large-scale pressure dilatation and $\mathrm{vdW}$ stress-strain, respectively. Note that these two terms contain no modes at small scales $<\ell$. Therefore they contribute only to the conversion of the large-scale kinetic energy into internal energy and vice versa. The third term on the right-hand side of (16) denotes the scale-to-scale kinetic energy flux (11). The definitions and physical meanings of each term comprising $Q_{\ell}^{\text {flux }}$ are given as follows. Deformation work is defined by

$$
\Pi_{\ell}:=-\bar{\rho}_{\ell} \nabla \tilde{\mathbf{v}}_{\ell}: \tilde{\tau}_{\ell}(\mathbf{v}, \mathbf{v}),
$$

where $\tilde{\tau}_{\ell}(\mathbf{v}, \mathbf{v}):=(\widetilde{\mathbf{v v}})_{\ell}-\tilde{\mathbf{v}}_{\ell} \tilde{\mathbf{v}}_{\ell}$, and it represents the work done by the large-scale $(>\ell)$ strain $\nabla \tilde{\mathbf{v}}_{\ell}$ against the smallscale $(<\ell)$ stress $\bar{\rho}_{\ell} \tilde{\tau}_{\ell}(\mathbf{v}, \mathbf{v})$. Baropycnal work is defined by

$$
\Lambda_{\ell}^{(p)}:=\frac{1}{\bar{\rho}_{\ell}} \nabla \bar{p}_{\ell} \cdot \bar{\tau}_{\ell}(\rho, \mathbf{v}),
$$

where $\bar{\tau}_{\ell}(\rho, \mathbf{v}):=\overline{(\rho \mathbf{v}}_{\ell}-\bar{\rho}_{\ell} \overline{\mathbf{v}}_{\ell}$, and it represents the work done by the large-scale pressure gradient force $-\nabla \bar{p}_{\ell} / \bar{\rho}_{\ell}$ against the small-scale mass flux $\bar{\tau}_{\ell}(\rho, \mathbf{v})$. Capillary work, which has a form similar to that of baropycnal work,

$$
\Lambda_{\ell}^{(\Sigma)}:=\frac{1}{\bar{\rho}_{\ell}} \nabla \cdot \overline{\boldsymbol{\Sigma}}_{\ell} \cdot \bar{\tau}_{\ell}(\rho, \mathbf{v}),
$$

represents the work done by the large-scale force $\nabla \cdot \overline{\boldsymbol{\Sigma}}_{\ell} / \bar{\rho}_{\ell}$ against the small-scale mass flux $\bar{\tau}_{\ell}(\rho, \mathbf{v})$. Note that in (16), only these three terms are capable of the direct transfer of kinetic energy across scales, because each of the three terms has a form "large-scale $(>\ell)$ quantity $\times$ small-scale $(<\ell)$ quantity," whereas the other terms on the right-hand side of (16) do not.

In the steady state, the spatial averaging of (16) gives

$$
\left\langle Q_{\ell}^{\text {flux }}\right\rangle=\left\langle\bar{p}_{\ell} \nabla \cdot \overline{\mathbf{v}}_{\ell}\right\rangle+\left\langle\overline{\boldsymbol{\Sigma}}_{\ell}: \nabla \overline{\mathbf{v}}_{\ell}\right\rangle-\left\langle D_{\ell}\right\rangle+\left\langle\epsilon_{\ell}^{\text {in }}\right\rangle .
$$

For the first term on the right-hand side, because $\langle p \nabla \cdot \mathbf{v}\rangle$ receives most of its contribution from scales $\gg \ell_{\text {large }}$, it can 
be approximated as $\left\langle\bar{p}_{\ell} \nabla \cdot \overline{\mathbf{v}}_{\ell}\right\rangle \approx\langle p \nabla \cdot \mathbf{v}\rangle$ for $\ell \ll \ell_{\text {large. }}$. Similarly, because the contribution to $\langle\boldsymbol{\Sigma}: \nabla \mathbf{v}\rangle$ from scales $\gg$ $\ell_{\text {small }}$ is negligible, the second term becomes $\left\langle\overline{\boldsymbol{\Sigma}}_{\ell}: \nabla \overline{\mathbf{v}}_{\ell}\right\rangle \approx 0$ for $\ell \gg \ell_{\text {small }}$. In addition, because the Kolmogorov scale is sufficiently smaller than other length scales and $\mathbf{f}$ acts at the large scale $L$, the viscous dissipation $\left\langle\bar{D}_{\ell}\right\rangle$ and energy injection $\left\langle\epsilon_{\ell}^{\text {in }}\right\rangle$ can be approximated as $\left\langle\bar{D}_{\ell}\right\rangle \approx 0$ and $\left\langle\epsilon_{\ell}^{\text {in }}\right\rangle \approx$ $\langle\mathbf{v} \cdot \mathbf{f}\rangle$ for $\ell_{\text {small }} \ll \ell \ll \ell_{\text {large }}$, respectively [27]. Thus, in the intermediate-scale range $\ell_{\text {small }} \ll \ell \ll \ell_{\text {large }}$, (20) becomes [50]

$$
\begin{aligned}
\left\langle Q_{\ell}^{\text {flux }}\right\rangle & \approx\langle p \nabla \cdot \mathbf{v}\rangle+\langle\mathbf{v} \cdot \mathbf{f}\rangle \\
& =\epsilon_{\mathrm{eff}} .
\end{aligned}
$$

Note that although the mean total scale-to-scale kinetic energy flux $\left\langle Q_{\ell}^{\text {flux }}\right\rangle$ is scale independent in the inertial range, the three terms $\left\langle\Pi_{\ell}\right\rangle,\left\langle\Lambda_{\ell}^{(p)}\right\rangle$, and $\left\langle\Lambda_{\ell}^{(\Sigma)}\right\rangle$ are not necessarily scale independent individually. In fact, because $\Sigma$ is appreciable at scales $\ll \ell_{c},\left\langle\Pi_{\ell}\right\rangle$ and $\left\langle\Lambda_{\ell}^{(p)}\right\rangle$ are dominant at scales $\gg \ell_{c}$, whereas $\left\langle\Lambda_{\ell}^{(\Sigma)}\right\rangle$ develops at scales $\ll \ell_{c}$. Therefore, (21) can be further rewritten as

$$
\epsilon_{\mathrm{eff}} \approx\left\langle Q_{\ell}^{\text {flux }}\right\rangle \approx\left\{\begin{array}{l}
\left\langle\Pi_{\ell}\right\rangle+\left\langle\Lambda_{\ell}^{(p)}\right\rangle \text { for } \ell_{c} \ll \ell \ll \ell_{\text {large }}, \\
\left\langle\Lambda_{\ell}^{(\Sigma)}\right\rangle \text { for } \ell_{\text {small }} \ll \ell \ll \ell_{c} .
\end{array}\right.
$$

We can also derive the result (22) more rigorously by evaluating the scale dependence of the energy fluxes using functional analysis [51].

We now consider the singularity of the velocity field that is necessary to satisfy (22). To this end, we investigate $\delta a(\ell):=$ $|\mathbf{a}(\mathbf{x}+\ell)-\mathbf{a}(\mathbf{x})|$ for a field $\mathbf{a}(\mathbf{x})$ using the assumption of homogeneity and isotropy. The following pointwise estimation is based on a more sophisticated analysis using Besov spaces [51]. In the range of $\ell_{c} \ll \ell \ll \ell_{\text {large }}$, the baropycnal work and deformation work are the main sources of the energy cascade. These two energy fluxes can be expressed in terms of increments:

$$
\begin{array}{r}
\Lambda_{\ell}^{(p)}=\frac{1}{\bar{\rho}_{\ell}} \nabla \bar{p}_{\ell} \cdot \bar{\tau}_{\ell}(\rho, \mathbf{v}) \sim \frac{\delta p(\ell) \delta \rho(\ell) \delta v(\ell)}{\rho_{0} \ell}, \\
\Pi_{\ell}=-\bar{\rho}_{\ell} \nabla \tilde{\mathbf{v}}_{\ell}: \tilde{\tau}_{\ell}(\mathbf{v}, \mathbf{v}) \sim-\frac{\rho_{0}(\delta v(\ell))^{3}}{\ell},
\end{array}
$$

where we have used $\bar{\rho}_{\ell} \sim \rho_{0}$ assuming homogeneity. We have also used an estimation that $\nabla \bar{f}_{\ell} \sim \delta f(\ell) / \ell$ and $\bar{\tau}_{\ell}(f, g) \sim$ $\delta f(\ell) \delta g(\ell)$, which can be made more rigorous using the $L^{p}$ norm [51]. From this expression it follows that $\left\langle\Lambda_{\ell}^{(p)}\right\rangle \rightarrow 0$ as $\ell \rightarrow 0$ because the density increment is bounded from above as $\delta \rho(\ell)=O(\ell)$, which holds for all $\ell>0$ because the entropy functional contains the density gradient term $\propto$ $|\nabla \rho|^{2}<\infty$. We therefore conclude that $\left\langle\Pi_{\ell}\right\rangle \approx \epsilon_{\text {eff }}$ for $\ell_{c} \ll$ $\ell \ll \ell_{\text {large }}$. Then, from the expression (24) we obtain $\delta v(\ell) \sim$ $\rho_{0}^{-1 / 3} \epsilon_{\mathrm{eff}}^{1 / 3} \ell^{1 / 3}$ in this scale range. This result implies that the velocity power spectrum $E^{v}(k)$ follows the Kolmogorov spectrum: $E^{v}(k) \sim \rho_{0}^{-2 / 3} \epsilon_{\mathrm{eff}}^{2 / 3} k^{-5 / 3}$ for $\ell_{\text {large }}^{-1} \ll k \ll \ell_{c}^{-1}$.

In the range of $\ell_{\text {small }} \ll \ell \ll \ell_{c}$, the energy transfer is dominated by capillary work. The capillary work can be expressed in terms of increments:

$$
\Lambda_{\ell}^{(\Sigma)}=\frac{1}{\bar{\rho}_{\ell}} \nabla \cdot \bar{\Sigma}_{\ell} \cdot \bar{\tau}_{\ell}(\rho, \mathbf{v}) \sim \frac{\bar{\Sigma}_{\ell} \delta \rho(\ell) \delta v(\ell)}{\rho_{0} \ell} .
$$

Here, note that we cannot naively estimate as in $\nabla \cdot \overline{\boldsymbol{\Sigma}}_{\ell} \sim$ $\delta \Sigma(\ell) / \ell$ because $\Sigma$ already contains higher-order derivatives of $\rho$. In this scale range, we must consider the density increment $\delta \rho(\ell)$ because density fluctuations are appreciable. The scale dependence of $\delta \rho(\ell)$ can be complicated because of the strong turbulent effect [52], although it may be bounded from above as $\delta \rho(\ell)=O(\ell)$. Here, as a first step to estimate the spectral exponent, we consider the consequence of imposing only the loose condition that $\delta \rho(\ell)=O(\ell)$. Then, by integrating by parts, we can estimate that $\bar{\Sigma}_{\ell} \delta \rho(\ell) \sim Z$, where $Z$ is a scale-independent quantity [51]. Hence, we conclude that $\delta v(\ell) \sim Z^{-1} \rho_{0} \epsilon_{\text {eff }} \ell$ and $E^{v}(k) \sim Z^{-2} \rho_{0}^{2} \epsilon_{\text {eff }}^{2} k^{-3}$ in this scale range.

Concluding remarks. In summary, we have shown that supercritical turbulence near a critical point can exhibit the van der Waals cascade. The interesting point here is that the results are similar to those reported for pure quantum turbulence [53]. This implies that pure quantum turbulence and van der Waals turbulence belong to the same "universality class." Therefore our results may also provide an interesting perspective from which to understand quantum turbulence, which will help illuminate the role of quantized vortices and Kelvin waves. Finally, we remark that there is a possibility that the spectrum $k^{-3}$ becomes shallower because of the depletion of nonlinearity [28,53-55] or the regularity of the temperature and density gradient fields [51].

The problem addressed in this Letter could lead to an understanding not only of turbulence but also of the relation between the macroscopic and microscopic descriptions of nature. We therefore hope that experiments will be conducted to verify our predictions.

Acknowledgments. T.T. was supported by JSPS KAKENHI Grant No. 20J20079, a Grant-in-Aid for JSPS Fellows. S.S. was supported by JSPS KAKENHI through Grants No. 17H01148, No. 19H05795, and No. 20K20425.
[1] U. Frisch, Turbulence (Cambridge University Press, Cambridge, England, 1995).

[2] L. F. Richardson, Weather Prediction by Numerical Process (Cambridge University Press, Cambridge, England, 1922).

[3] A. N. Kolmogorov, The local structure of turbulence in incompressible viscous fluid for very large Reynolds numbers, Dokl. Akad. Nauk SSSR 30, 301 (1941).
[4] A. N. Kolmogorov, Dissipation of energy in locally isotropic turbulence, Dokl. Akad. Nauk SSSR 32, 16 (1941).

[5] H. L. Swinney and D. L. Henry, Dynamics of fluids near the critical point: Decay rate of order-parameter fluctuations, Phys. Rev. A 8, 2586 (1973).

[6] D. S. Cannell, Measurement of the long-range correlation length of $\mathrm{SF}_{6}$ very near the critical point, Phys. Rev. A 12, 225 (1975). 
[7] M. Assenheimer and V. Steinberg, Rayleigh-Bénard Convection near the Gas-Liquid Critical Point, Phys. Rev. Lett. 70, 3888 (1993).

[8] S. Ashkenazi and V. Steinberg, High Rayleigh Number Turbulent Convection in a Gas Near the Gas-Liquid Critical Point, Phys. Rev. Lett. 83, 3641 (1999).

[9] S. Ashkenazi and V. Steinberg, Spectra and Statistics of Velocity and Temperature Fluctuations in Turbulent Convection, Phys. Rev. Lett. 83, 4760 (1999).

[10] G. Accary, P. Bontoux, and B. Zappoli, Turbulent RayleighBénard convection in a near-critical fluid by three-dimensional direct numerical simulation, J. Fluid Mech. 619, 127 (2009).

[11] J. D. van der Waals, The thermodynamic theory of capillarity under the hypothesis of a continuous variation of density, J. Stat. Phys. 20, 200 (1979), original work published 1893; translated by J. S. Rowlinson.

[12] V. L. Ginzburg and L. D. Landau, On the theory of superconductivity, Zh. Eksp. Teor. Fiz. 20, 1064 (1950).

[13] J. W. Cahn and J. E. Hilliard, Free energy of a nonuniform system. I. Interfacial free energy, J. Chem. Phys 28, 258 (1958).

[14] D. J. Korteweg, Sur la forme que prennent les équations du mouvements des fluides si l'ons tient compte des forces capillaires causées par des variations de densité, Arch. Néerl. Sci. Exactes Nat. 6, 1 (1901).

[15] J. E. Dunn and J. Serrin, On the thermomechanics of interstitial working, in The Breadth and Depth of Continuum Mechanics (Springer, New York, 1986), pp. 705-743.

[16] A. Onuki, Dynamic van der Waals Theory of Two-Phase Fluids in Heat Flow, Phys. Rev. Lett. 94, 054501 (2005).

[17] A. Onuki, Dynamic van der Waals theory, Phys. Rev. E 75, 036304 (2007).

[18] L. Onsager, Statistical hydrodynamics, Nuovo Cimento Suppl. 6, 279 (1949).

[19] G. L. Eyink, Review of the Onsager "ideal turbulence" theory, arXiv: 1803.02223.

[20] G. L. Eyink and K. R. Sreenivasan, Onsager and the theory of hydrodynamic turbulence, Rev. Mod. Phys. 78, 87 (2006).

[21] G. I. Taylor, Observations and speculations on the nature of turbulent motion, in Scientific Papers, Vol. 2, edited by G. K. Batchelor (Cambridge University Press, Cambridge, England, 1917), p. 69.

[22] E. Feireisl, P. Gwiazda, A. Świerczewska-Gwiazda, and E. Wiedemann, Regularity and energy conservation for the compressible Euler equations, Arch. Ration. Mech. An. 223, 1375 (2017).

[23] G. L. Eyink and T. D. Drivas, Cascades and Dissipative Anomalies in Compressible Fluid Turbulence, Phys. Rev. X 8, 011022 (2018).

[24] T. D. Drivas and G. L. Eyink, An Onsager singularity theorem for turbulent solutions of compressible Euler equations, Commun. Math. Phys. 359, 733 (2018).

[25] H. Aluie, Compressible Turbulence: The Cascade and Its Locality, Phys. Rev. Lett. 106, 174502 (2011).

[26] H. Aluie, Scale locality and the inertial range in compressible turbulence, arXiv:1101.0150.

[27] H. Aluie, Scale decomposition in compressible turbulence, Physica D 247, 54 (2013).

[28] G. L. Eyink, Cascades and Dissipative Anomalies in Nearly Collisionless Plasma Turbulence, Phys. Rev. X 8, 041020 (2018).
[29] C. De Lellis and L. Székelyhidi, Dissipative continuous Euler flows, Invent. Math. 193, 377 (2013).

[30] P. Isett, A proof of Onsager's conjecture, Ann. Math. 188, 871 (2018).

[31] T. Buckmaster, C. De Lellis, L. Székelyhidi, and V. Vicol, Onsager's conjecture for admissible weak solutions, Commun. Pure Appl. Math. 72, 229 (2019).

[32] T. Buckmaster and V. Vicol, Convex integration and phenomenologies in turbulence, arXiv:1901.09023.

[33] C. De Lellis and L. Székelyhidi, Jr., On turbulence and geometry: From Nash to Onsager, arXiv:1901.02318.

[34] A. Onuki, Phase Transition Dynamics (Cambridge University Press, Cambridge, England, 2002).

[35] Here we have assumed that the gradient contribution to the internal energy vanishes [17].

[36] See Supplemental Material at http://link.aps.org/supplemental/ 10.1103/PhysRevResearch.3.L032027 for the derivation of the pressure tensor.

[37] D. M. Anderson, G. B. McFadden, and A. A. Wheeler, Diffuseinterface methods in fluid mechanics, Annu. Rev. Fluid Mech. 30, 139 (1998).

[38] S. Benzoni-Gavage, Propagating phase boundaries and capillary fluids, Lecture notes of CIRM Summer School "Mathematical Fluid Dynamics" (2010), available at http://math.univlyon1.fr/ benzoni/Levico.pdf.

[39] For the Euler-Korteweg equations, an Onsager-type condition was obtained in [56].

[40] A. Onuki, Dynamic equations and bulk viscosity near the gas-liquid critical point, Phys. Rev. E 55, 403 (1997).

[41] See Supplemental Material at http://link.aps.org/supplemental/ 10.1103/PhysRevResearch.3.L032027 for the derivation of the equilibrium correlation length.

[42] H. Aluie, S. Li, and H. Li, Conservative cascade of kinetic energy in compressible turbulence, Astrophys. J. Lett. 751, L29 (2012).

[43] J. Wang, Y. Yang, Y. Shi, Z. Xiao, X. T. He, and S. Chen, Cascade of Kinetic Energy in Three-Dimensional Compressible Turbulence, Phys. Rev. Lett. 110, 214505 (2013).

[44] H. Tennekes and J. L. Lumley, A First Course in Turbulence (MIT Press, Cambridge, MA, 1972).

[45] A. Lees and H. Aluie, Baropycnal work: A mechanism for energy transfer across scales, Fluids 4, 92 (2019).

[46] See Supplemental Material at http://link.aps.org/supplemental/ 10.1103/PhysRevResearch.3.L032027 for scale locality of the van der Waals cascade.

[47] P. Nikolai, B. Rabiyat, A. Aslan, and A. Ilmutdin, Supercritical $\mathrm{CO}_{2}$ : Properties and technological applications-A review, J. Therm. Sci. 28, 394 (2019).

[48] P. Carlès, A brief review of the thermophysical properties of supercritical fluids, J. Supercrit. Fluids 53, 2 (2010).

[49] V. Vesovic, W. A. Wakeham, G. A. Olchowy, J. V. Sengers, J. T. R. Watson, and J. Millat, The transport properties of carbon dioxide, J. Phys. Chem. Ref. Data 19, 763 (1990).

[50] See Supplemental Material at http://link.aps.org/supplemental/ 10.1103/PhysRevResearch.3.L032027 for the detailed derivation of (21). 
[51] See Supplemental Material at http://link.aps.org/supplemental/ 10.1103/PhysRevResearch.3.L032027 for detailed calculations and explanations.

[52] M. Hnatič, G. Kalagov, and M. Nalimov, Turbulent mixing of a critical fluid: The non-perturbative renormalization, Nucl. Phys. B 926, 1 (2018).

[53] T. Tanogami, Theoretical analysis of quantum turbulence using the Onsager ideal turbulence theory, Phys. Rev. E 103, 023106 (2021).
[54] S. Boldyrev, On the spectrum of magnetohydrodynamic turbulence, Astrophys. J. Lett. 626, L37 (2005).

[55] J. Mason, F. Cattaneo, and S. Boldyrev, Dynamic Alignment in Driven Magnetohydrodynamic Turbulence, Phys. Rev. Lett. 97, 255002 (2006).

[56] T. Dębiec, P. Gwiazda, A. Świerczewska-Gwiazda, and A. Tzavaras, Conservation of energy for the Euler-Korteweg equations, Calc. Var. Partial Differ. Equations 57, 160 (2018). 